\title{
Use of Recyclable Materials in Sustainable Civil Engineering Applications
}

\author{
Monica Prezzi, ${ }^{1}$ Paola Bandini, ${ }^{2}$ J. Antonio H. Carraro, ${ }^{3}$ and Paulo J. M. Monteiro ${ }^{4}$ \\ ${ }^{1}$ Civil Engineering Department, Purdue University, West Lafayette, IN 47907, USA \\ ${ }^{2}$ Civil Engineering Department, New Mexico State University, Las Cruces, NM 88003-8001, USA \\ ${ }^{3}$ Centre for Offshore Foundation Systems M053, The University of Western Australia, 35 Stirling Highway, Crawley, WA 6009, Australia \\ ${ }^{4}$ Civil and Environmental Engineering, University of California Berkeley, Berkeley, CA 94720, USA \\ Correspondence should be addressed to Monica Prezzi, mprezzi@purdue.edu
}

Received 15 November 2011; Accepted 15 November 2011

Copyright (C) 2011 Monica Prezzi et al. This is an open access article distributed under the Creative Commons Attribution License, which permits unrestricted use, distribution, and reproduction in any medium, provided the original work is properly cited.

Various types of recyclable materials are currently used in civil engineering applications. These include tire shreds, ground tire rubber, fly and bottom ash, blast-furnace slag, steel slag, cement kiln dust, silica fume, crushed glass, reclaimed asphalt pavement (RAP), and rice husk ash. Reutilization of these recyclable materials is especially beneficial in civil engineering applications that require large volumes of materials. When these waste products are used in place of other conventional materials, natural resources and energy are preserved and expensive and/or potentially harmful waste disposal is avoided. This special issue deals with the use of recyclable materials in diverse civil engineering applications focusing on sustainable development. The papers in this special issue present results of laboratory tests and important research findings for these materials, recommendations for debris recycling practices, and documented field applications of several waste or recyclable materials.

The paper by D. L. Brandon et al. deals with the Hurricane Katrina storm debris removal practices in 16 southern Mississippi counties in the USA and the associated recycling efforts. Various types of debris, which included vegetation, construction material, electronic waste, vehicles, and vessels, were removed from public and private property. The scope within the respective counties varied from removal of vegetation only to removal of every eligible form of debris. The recommendations proposed in this paper will help improve planning and implementation of recycling efforts during debris removal missions in the future.

The paper by S. Talukdar et al. investigated the use of waste materials, such as crushed glass, ground tire rubber, and recycled aggregate, in concrete. Test results demonstrated that ground tire rubber introduced significant amounts of air into the mixtures tested, affecting adversely their compressive strength. The air introduced into these mixtures was partly removed by using a defoamer, which was less effective for mixtures containing recycled aggregates, glass, and ground tire rubber. Freeze-thaw test results showed that this air entrained in the mixtures was not able to improve their freeze-thaw resistance. The authors indicated that further research is needed to optimize the design and performance of lightweight, low-carbon footprint concrete materials.

The paper by I. Z. Yildirim and M. Prezzi provides an overview of the different types of steel slag generated from basic-oxygen-furnace steelmaking, electric-arc-furnace steelmaking, and ladle-furnace steel refining processes. The mineralogical and morphological properties of basic-oxygenfurnace and electric-arc-furnace-ladle slag samples generated from two steel plants in Indiana, USA are presented and discussed in detail based on results from X-Ray Diffraction analyses and Scanning Electron Microscopy studies.

The paper by C. E. Riley et al. presents the results of a laboratory-testing program to investigate the use of spray dryer ash as the primary or sole binding component in mortar for various applications. The study focuses on very high contents of spray dryer ash in a hardened product. The spray dryer ash, also known as spray dryer absorber, has selfcementing properties and is a byproduct of coal combustion and flue gas scrubbing processes. The test results showed that the spray dryer ash mixed with water along or with recycled polymer fibers has very low strength and properties 
that are not suitable for structural engineering applications. However, the addition of very small percentages of Portland cement and recycled fibers increased the compressive and flexural strengths. The experimental results showed that spray dryer ash has potential for beneficial use in construction and material applications.

The paper by J. W. van de Lindt and R. K. Rechan of this special issue presents the method and results of experiments carried out to study the seismic behavior of a concrete portal frame with fifty percent of its cement content replaced by a spray dryer ash (SDA). Based on multiple shake table tests, the high-content SDA frame was found to perform as well as the standard concrete frame for two earthquakes exceeding design-level intensity earthquakes.

Another paper entitled "Cellular fibroma of the ovary with multiloculated macroscopic characteristics: a case report" investigated the use of recycled aggregate concrete made with coarse aggregate from the demolition of a concrete structure. The experimental research compared the compressive strength, elastic modulus, and drying shrinkage of concrete made with a reference aggregate and concrete containing $30 \%$ of recycled aggregate. The results indicated that the recycled aggregate concrete can meet the requirements for structural concrete up to C32/40 strength class.

There is a paper that provides a critical review of sustainable construction with emphasis on the use of concrete in the region covered by the Gulf Cooperation Council. Members of this Council include Persian Gulf States of Bahrain, Kuwait, Oman, Qatar, Saudi Arabia, and the United Arab Emirates. The authors analyzed the existing literature on the use of recycled aggregate for the stringent conditions of the Gulf region. The analysis indicated that while there is research on recycled aggregate concrete in laboratory conditions, the technology transfer to field conditions has been small. Another limitation identified by the authors is that most of the research has focused on the mechanical properties of the recycled aggregate with limited emphasis on the durability.

The paper entitled "Use of reclaimed asphalt pavement in conjunction with ground improvement: a case history" describes the use of RAP and geosynthetic ground improvement in a design-built project for the reconstruction of an asphalt parking lot. The project was successfully completed at a cost significantly lower than the original cut and replace specification. The field observations during this project regarding drainage of the RAP aggregate base are in agreement with prior research findings in the literature and suggest that RAP aggregate base courses can be constructed with properties comparable to virgin stone aggregate base courses compacted with similar conditions. The paper also discusses contractual issues, such as the risk of adopting a less conventional method and materials to reduce construction costs and the efficiencies of the design-built approach in those cases.

Monica Prezzi

Paola Bandini

J. Antonio H. Carraro

Paulo J. M. Monteiro 

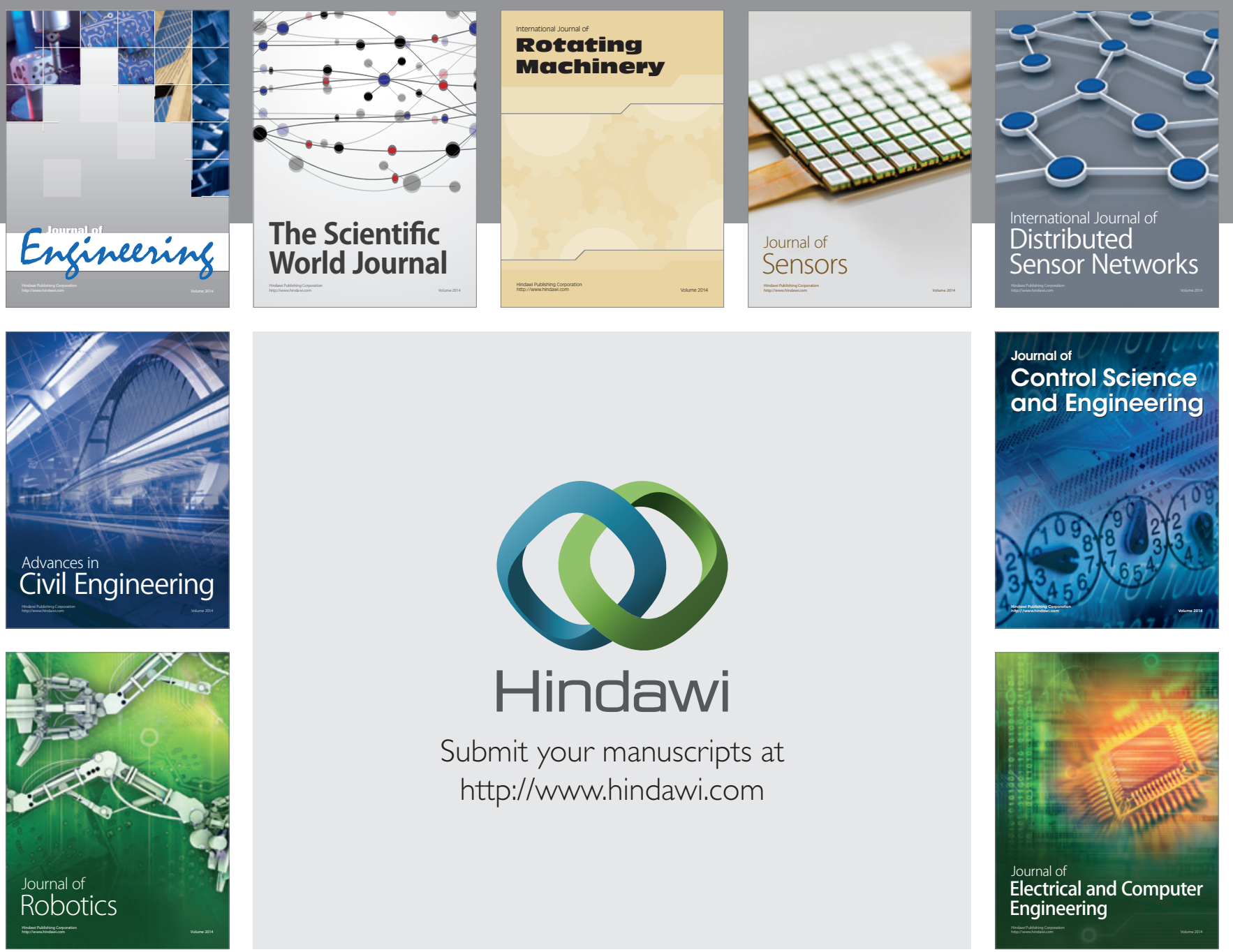

Submit your manuscripts at

http://www.hindawi.com
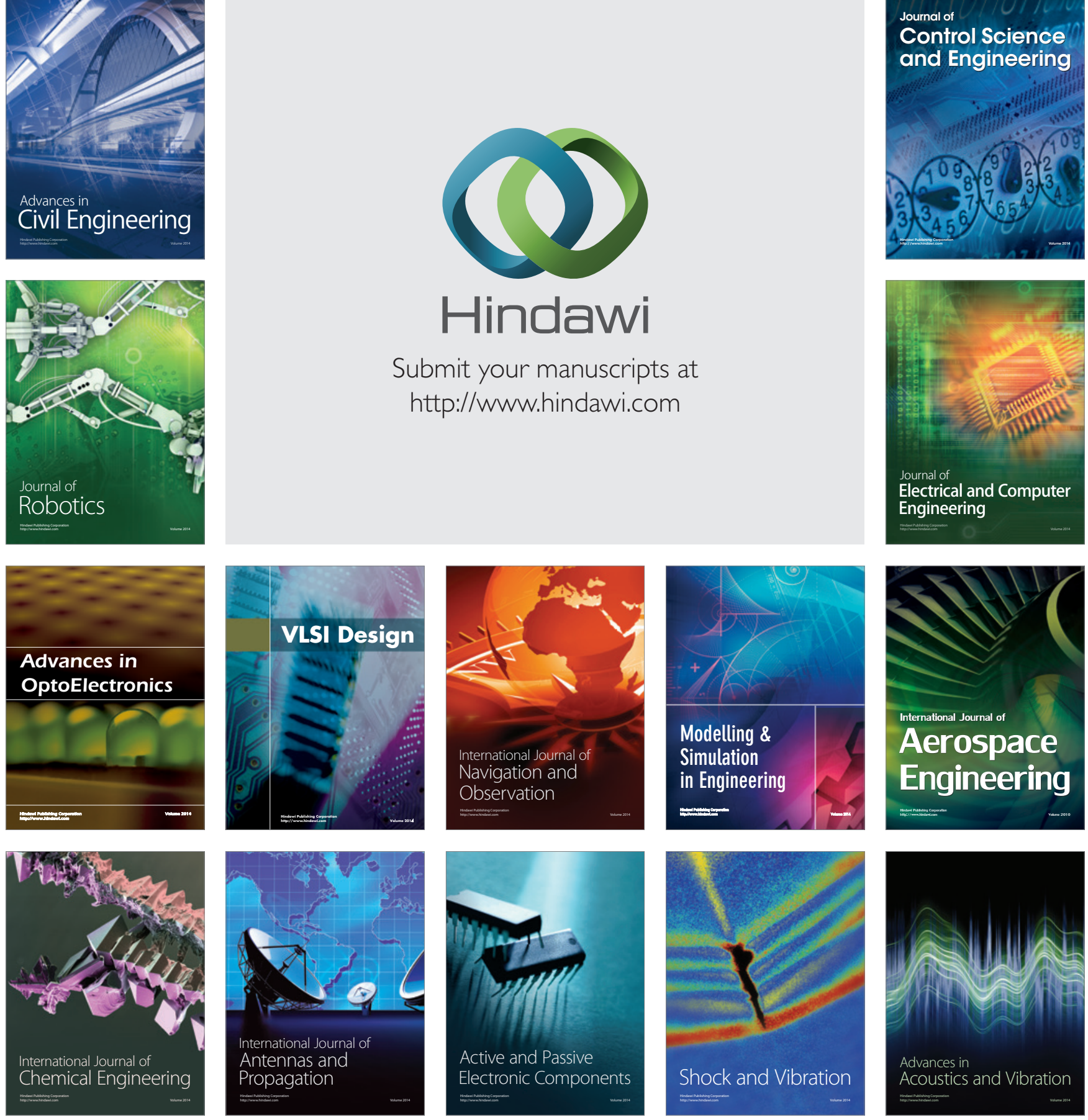